\title{
Flow of a Blood Analogue Solution Through Microfabricated Hyperbolic Contractions
}

\author{
P.C. Sousa, I.S. Pinho, F.T. Pinho, M.S.N. Oliveira, and M.A. Alves
}

\begin{abstract}
The flow of a blood analogue solution past a microfabricated hyperbolic contraction followed by an abrupt expansion was investigated experimentally. The shape of the contraction was designed in order to impose a nearly constant strain rate to the fluid along the centerline of the microgeometry. The flow patterns of the blood analogue solution and of a Newtonian reference fluid (deionized water), captured using streak line imaging, are quite distinct and illustrate the complex behavior of the blood analogue solution flowing through the microgeometry. The flow of the blood analogue solution shows elastic-driven effects with vortical structures emerging upstream of the contraction, which are absent in Newtonian fluid flow. In both cases the flow also develops instabilities downstream of the expansion but these are inertia driven. Therefore, for the blood analogue solution at high flow rates the competing effects of inertia and elasticity lead to complex flow patterns and unstable flow develops.
\end{abstract}

Keywords Blood analogue fluid - Extensional flow · Microfluidics · Viscoelasticity · Flow visualization · Hyperbolic contraction

\section{Introduction}

The Human blood is a complex fluid consisting of a suspension of cellular elements, $98 \%$ of which are erythrocytes (red blood cells, RBCs) suspended in an aqueous matrix, the plasma, containing also proteins and other solutes. In healthy human

P.C. Sousa, I.S. Pinho, M.S.N. Oliveira (凶), and M.A. Alves

Faculdade de Engenharia da Universidade do Porto, Departamento de Engenharia Química, Centro de Estudos de Fenómenos de Transporte, Rua Dr. Roberto Frias, 4200-465 Porto, Portugal e-mail: deq06010@fe.up.pt; bio06019@fe.up.pt; monica.oliveira@fe.up.pt; mmalves@fe.up.pt

F.T. Pinho

Faculdade de Engenharia da Universidade do Porto, Centro de Estudos de Fenómenos de Transporte, Departamento de Engenharia Mecânica, Rua Dr. Roberto Frias, 4200-465 Porto, Portugal e-mail: fpinho@fe.up.pt 
blood the white blood cells (leukocytes) and platelets represent about $2 \%$ of the suspended cellular elements. The ratio between the volume of the RBCs and the total volume of blood is the so-called hematocrit, an important physiological parameter to be considered in the characterization of human blood. Although we can consider plasma as a Newtonian fluid, the deformable RBCs and its structures confer nonNewtonian properties to this rheologically complex fluid. In effect, at low shear rates RBCs may form aggregated structures, named rouleaux, the dynamics of which impact severely on blood rheology [19]. The aggregation of blood cells depends on the protein concentration in plasma (a good example that minor amounts of an additive can have a large impact in fluid structure and rheology) and on the shear rate and is a reversible process that leads to a viscoelastic, shear-thinning and thixotropic behavior of blood $[16,17]$. As such, blood shear viscosity depends on a number of variables, namely, hematocrit, the shear rate and even the vessel diameter, the latter known as the Fåhraeus-Lindqvist effect [10].

Despite the wealth of information on the complex rheology of blood [10,17], the vast majority of research on hemodynamics assumes that blood behaves as a Newtonian fluid. This can be acceptable when blood is flowing through major arteries [5] however, non-Newtonian characteristics become important in small vessels or when the characteristic times of the flow and fluid become comparable, and in this respect it is also important to realize that the flow is time-dependent essentially everywhere. Furthermore, viscoelastic effects are enhanced when the vessel geometry exhibits fluid features such as contractions/expansions (e.g. due to fatty deposits), taper or bifurcations in addition to local time dependency. One such relevant feature is the stenosis, a local constriction of a blood vessel, usually defined as the percentage of vessel diameter reduction, due to the adherence and accumulation of cells, especially of fatty material. This accumulative process has a shear-rate dependent positive feedback mechanism, since more deposition takes place at higher shear rates and shear rates increase as the diameter of the vessel is reduced. Studies reported so far show that flow separation occurs in the expansion region of the stenosis at Reynolds numbers on the order of 10 for a $70 \%$ stenosis [5].

Since the use of blood in experimental studies is not always practical, primarily due to safety reasons, viscoelastic fluids with rheological characteristics similar to human blood are a good and safe alternative. Vlastos et al. [16] studied the combination of steady and oscillatory shear on the human blood flow behavior. For this purpose, they also used blood analogue solutions made from high molecular weight polymers dissolved in water, namely aqueous solutions of polyacrylamide and xanthan gum at different concentrations. The polyacrylamide and xanthan gum solutions have a rheological behavior similar to that of human blood at concentrations of 125 and $500 \mathrm{ppm}$ (w/w), respectively, especially at low shear rates whereas at high shear rates they tend to exhibit higher viscosity and elasticity than blood. Still, these are adequate analogue fluids in a variety of flow conditions.

In this work, we compare the flows of a Newtonian fluid and a viscoelastic blood analogue through microfluidic contraction/expansion geometries to highlight the non-Newtonian effects at the microscale. The Newtonian fluid is water and the blood analogue is an aqueous xanthan gum solution $(500 \mathrm{ppm} w / \mathrm{w})$ as proposed in $[14,16]$. 


\section{Experimental}

\subsection{Microchannel Geometry}

In this study three different microgeometries were studied. In all cases, the microchannels used are planar and have a contraction with a hyperbolic shape, followed by an abrupt expansion. The hyperbolic shape was chosen in order to provide a nearly constant strain rate of the fluid flow along the centerline of the microgeometry, as proposed by Oliveira et al. [8]. Using this configuration, it is possible to study the response of the fluid under strong accelerations, for controlled extensional flow conditions.

The geometry and length scale of the microchannels studied are comparable to small human vessels with a constriction. In the human circulatory system, capillaries are the smallest blood vessels, having diameters of 4-8 $\mu \mathrm{m}$ and arterioles typically have internal diameters in the range from 50 up to $100 \mu \mathrm{m}$ [2]. In Fig. 1 we show a microscopy image of a typical contraction-expansion geometry used in this study.

The total width, $D_{1}=400 \mu \mathrm{m}$ of the microchannels was kept constant for all geometries. The minimum width of the contraction, $D_{2}$, and the hyperbolic contraction length, $L_{\mathrm{c}}$ were varied in order to obtain different values of the total Hencky strain $\left(\varepsilon_{H}\right)$, here defined as $\varepsilon_{H}=\ln \left(D_{1} / D_{2}\right)$. The depth, $h$, of the microchannels used varied slightly relative to the nominal value of $50 \mu \mathrm{m}$ due to inaccuracies inherent to the fabrication procedure. In Table 1 we present the dimensions for each geometry.

The channels were fabricated in polydimethylsiloxane, PDMS (Sylgard 184, Dow Corning), from an SU-8 photoresist mold using standard soft lithography

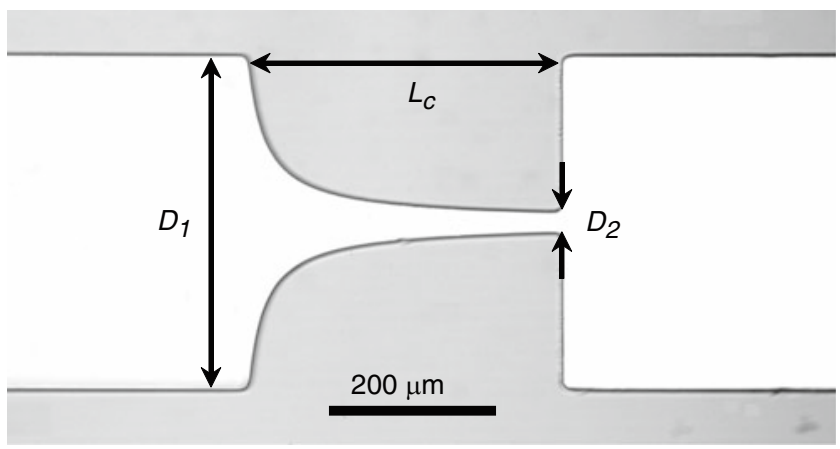

Fig. 1 Microchannel $S_{3}$ used in the experiments

Table 1 Geometry

dimensions and corresponding Hencky strain

\begin{tabular}{lllcl}
\hline Channel & $\varepsilon_{H}$ & $D_{2}(\mu \mathrm{m})$ & $L_{\mathrm{c}}(\mu \mathrm{m})$ & $h(\mu \mathrm{m})$ \\
\hline$S_{1}$ & 0.76 & 187 & 34 & 47 \\
$S_{2}$ & 1.86 & 62.4 & 128 & 45 \\
$S_{3}$ & 2.75 & 25.5 & 380 & 44 \\
\hline
\end{tabular}


techniques [6]. PDMS was utilized due to its transparency that permits optical access to the flow region, and also due to its simple and well-established fabrication techniques, which allow us to obtain geometries with well-defined features.

\subsection{Flow Visualization}

Visualizations of the flow patterns were carried out at room temperature, for a wide range of flow rates, using streak line photography. The optical setup consists of an inverted epi-fluorescence microscope (DMI 5000M, Leica Microsystems $\mathrm{GmbH}$ ) equipped with a CCD camera (DFC350 FX, Leica Microsystems GmbH), a light source (100 W mercury lamp) and a filter cube (Leica Microsystems GmbH, excitation filter BP 530-545 nm, dichroic $565 \mathrm{~nm}$ and barrier filter 610-675 nm). A syringe pump (PHD2000, Harvard Apparatus) was used to inject the fluid and control the flow rate in the microchannel. Syringes with different volumes $(50 \mu 1-$ $10 \mathrm{ml}$ ) were used according to the desired flow rate and connected to the microgeometries using Tygon tubing. The fluids were seeded with $1 \mu \mathrm{m}$ fluorescent tracer particles (Nile Red, Molecular Probes, Invitrogen, Ex/Em: 520/580 nm) and sodium dodecyl sulfate $(0.1 \mathrm{wt} \%$, Sigma-Aldrich) was added in order to minimize adhesion of fluorescent tracer particles to the channels walls. The microgeometries containing the seeded fluid were continuously illuminated and the light emitted by the fluorescent tracer particles was imaged through the microscope objective $(\times 10, \mathrm{NA}=0.25)$ onto the CCD array of the camera using "long" exposure times $(\sim 1 s)$ in order to capture the particle pathlines. All streak line images presented here are centered at the mid-plane of the microchannel.

\subsection{Rheological Characterization}

The viscoelastic fluid used in the experiments was an aqueous solution of xanthan gum (Sigma-Aldrich) with a concentration of $500 \mathrm{ppm} \mathrm{w} / \mathrm{w}$, which is a well established blood analogue solution, known to have a shear rheology behavior similar to that of human blood $[14,16]$. Deionized water was also used for comparison purposes. The shear viscosity and density of deionized water at $293.2 \mathrm{~K}$ are $1.003 \times 10^{-3} \mathrm{~Pa} \mathrm{~s}$ and $998.3 \mathrm{~kg} \mathrm{~m}^{-3}$, respectively. The density of the viscoelastic solution was measured at $293.2 \mathrm{~K}$ using density flasks $\left(\rho=998.8 \mathrm{~kg} \mathrm{~m}^{-3}\right)$.

The steady shear rheology of the xanthan gum (XG) solution was measured with a shear rheometer (Anton Paar, model Physica MCR301) using a cone-plate geometry $\left(75 \mathrm{~mm}, 1^{\circ}\right.$ angle) under shear rate control. Figure 2 compares the measured shear viscosity of the XG solution at $293.2 \mathrm{~K}$ with reported values for human blood at $310.2 \mathrm{~K}$ [15].

In Fig. 2 we also show as dashed lines the lower and upper limits that should be considered for accurate measurements of the shear viscosity. The lower boundary 


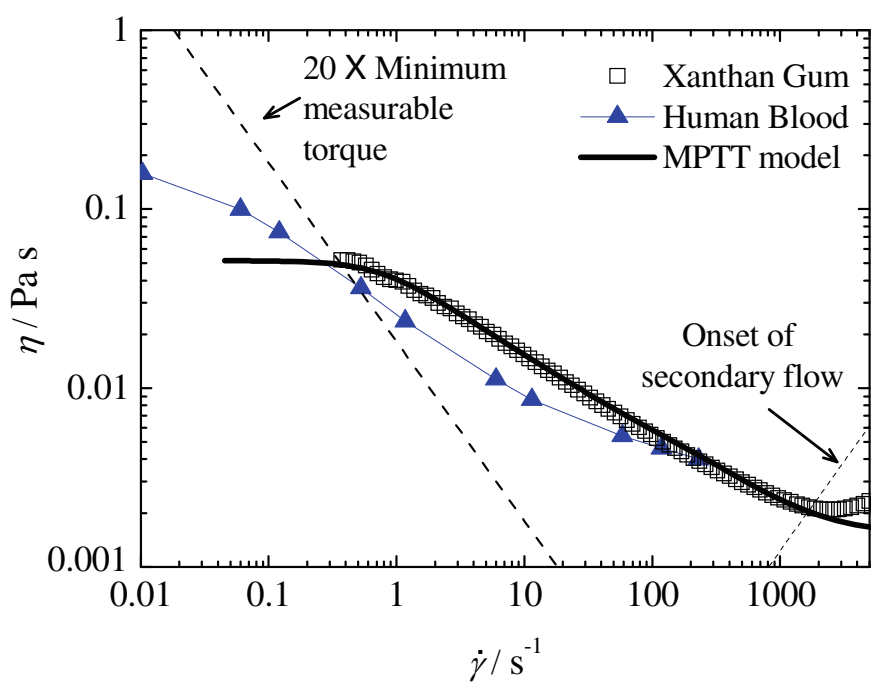

Fig. 2 Comparison of steady shear data of the xanthan gum solution at $293.2 \mathrm{~K}$ with reported values for human blood at $310.2 \mathrm{~K}$ [15]

of the shear viscosity was calculated considering 20 times the minimum resolvable torque specifications of the shear rheometer [1],

$$
\eta(\dot{\gamma})=\frac{3\left(20 \widetilde{s}_{\min }\right)}{2 \pi R^{3}} \frac{1}{\dot{\gamma}},
$$

where $\eta$ is the shear viscosity, $\dot{\gamma}$ is the shear rate, $\mathfrak{I}_{\min }$ is the rheometer minimum resolvable torque $\left(10^{-7} \mathrm{~N} \mathrm{~m}\right)$ and $R$ is the radius of the cone geometry used in the measurements.

The laminar flow of a Newtonian fluid between a rotating cone and a fixed plate is a purely rotational steady shear flow when the following relation is valid [13]:

$$
\frac{\omega \alpha^{2} \rho R^{2}}{12 \eta}<0.5
$$

where $\omega$ is the angular velocity and $\alpha$ is the angle between the cone and the plate. Therefore, the upper limit of the measurable shear viscosity, based on the onset of inertial instabilities (Taylor vortices) is given by

$$
\eta=\frac{\omega \alpha^{2} \rho R^{2}}{6} .
$$

Additionally, the shear viscosity curve was fitted using a modified Phan-ThienTanner model (MPTT) [18] with a Newtonian solvent contribution. The fit is also 
represented in Fig. 2. In this particular model, the total stress is $\boldsymbol{\sigma}=\boldsymbol{\tau}+\boldsymbol{\tau}_{\mathrm{s}}-p \mathbf{I}$, where $p$ is the pressure and $\tau_{\mathrm{s}}$ is the contribution of the Newtonian solvent given by $\boldsymbol{\tau}_{\mathrm{s}}=\eta_{\text {solvent }}\left(\nabla \mathbf{u}+\nabla \mathbf{u}^{\mathrm{T}}\right)$.

For an MPTT fluid, the constitutive equation for the polymer contribution $(\tau)$ to the extra stress tensor is given by,

$$
f(\operatorname{Tr} \boldsymbol{\tau}) \boldsymbol{\tau}+\lambda\left[\frac{\partial \tau}{\partial t}+\nabla \cdot \mathbf{u} \boldsymbol{\tau}\right]=\eta_{\text {polymer }}\left(\nabla \mathbf{u}+\nabla \mathbf{u}^{\mathrm{T}}\right)+\lambda\left(\boldsymbol{\tau} \cdot \nabla \mathbf{u}+\nabla \mathbf{u}^{\mathrm{T}} \cdot \boldsymbol{\tau}\right)
$$

Since we are using the linear form of the PTT model [11], $f(\operatorname{Tr} \tau)$ becomes:

$$
f(\operatorname{Tr} \tau)=1+\frac{\lambda \varepsilon}{\eta_{\text {polymer }}} \operatorname{Tr}(\tau)
$$

where $\varepsilon$ is the extensibility parameter and $\eta_{\text {polymer }}$ is the viscosity coefficient of the model, which is here given by a Carreau-type equation [18]:

$$
\eta_{\text {polymer }}=\frac{\eta_{\mathrm{p}}}{\left[1+(\Gamma \dot{\gamma})^{2}\right]^{\frac{1-n}{2}}}
$$

In Eq. $6, \Gamma$ is a time parameter, $n$ is the power-law index, $\eta_{\mathrm{p}}$ is the zero-shear-rate polymer viscosity and the shear-rate, $\dot{\gamma}$, is an invariant of the rate of deformation tensor $\left(\dot{\gamma}=\sqrt{(\dot{\gamma}: \dot{\gamma}) / 2}\right.$ with $\left.\dot{\gamma}=\nabla \mathbf{u}+\nabla \mathbf{u}^{\mathrm{T}}\right)$.

The fitted viscosity is thus given by $\eta=\left(\tau+\tau_{\text {solvent }}\right) / \dot{\gamma}$, where the parameters are: $\varepsilon=0.05, \eta_{\mathrm{p}}=0.05 \mathrm{Pas}, \Gamma=1.3 \mathrm{~s}, n=0.5$ and the solvent viscosity is $\eta_{\text {solvent }}=0.0015 \mathrm{Pas}$. The relaxation time considered in the fitting is the value determined using the CaBER measurements $(\lambda=3.77 \mathrm{~ms})$ described below. The fit to the experimental shear data was done in order to allow for the calculations of the shear viscosity and possibly other rheological material functions that are a function of the shear rate, as well as to provide relevant information to be used in future numerical simulations of the flow of the blood analogue solution used in this study.

The relaxation time of the fluid was determined at $293.2 \mathrm{~K}$ under extensional flow conditions using a capillary-breakup extensional rheometer (Haake CaBER 1, Thermo Scientific). During CaBER measurements, the liquid is placed between two circular plates, which are separated by a distance $h_{\mathrm{i}}$, and then an axial step strain is imposed until a final height $\left(h_{\mathrm{f}}\right)$ is reached. Subsequently, the liquid thread is left to relax and breakup under the combined action of capillary and extensional viscoelastic forces. The diameter of the filament $D(t)$ is monitored as a function of time, $t$, as shown in Fig. 3 for the xanthan gum solution. The circular plates used were $D_{\mathrm{p}}=6 \mathrm{~mm}$ in diameter and the initial and final aspect ratios, defined as $\Lambda_{\mathrm{i}}=h_{\mathrm{i}} / D_{\mathrm{p}}$ and $\Lambda_{\mathrm{f}}=h_{\mathrm{f}} / D_{\mathrm{p}}$, were set as 0.33 and 1.39 , respectively. The relaxation time, $\lambda$, can be determined by fitting the experimental data in the linear region of $\log [D(\mathrm{t})]$ as a function of $t$, since an elasto-capillary balance [4] leads to the relation $D(t) / D_{0} \propto \exp [-t /(3 \lambda)]$, where $D_{0}$ is the diameter of the filament at time $t=0$. The average relaxation time obtained by CaBER measurements was $3.77 \mathrm{~ms}$. 


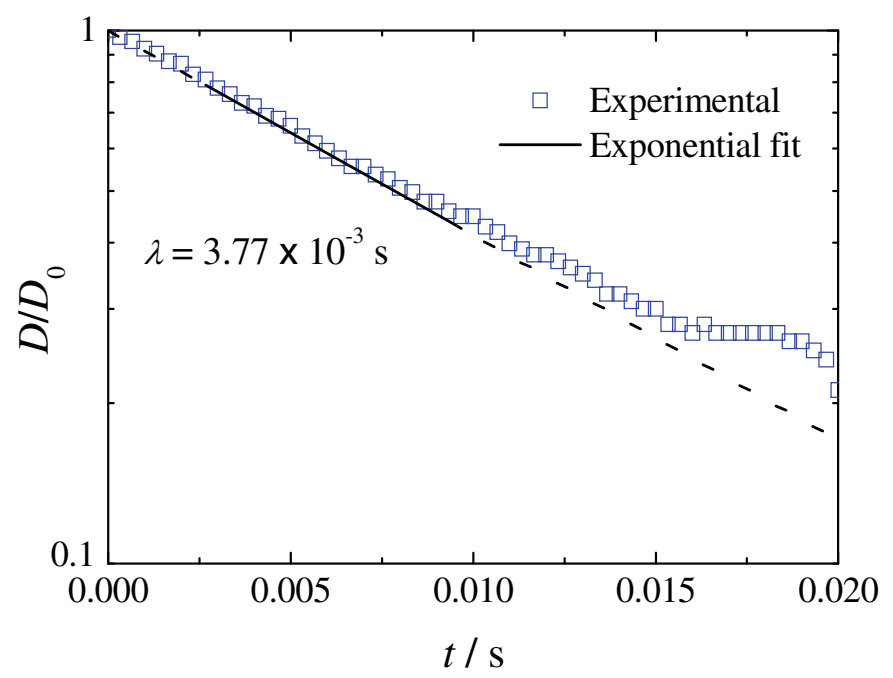

Fig. 3 Exponential decay of the xanthan gum filament diameter during a CaBER measurement at $293.2 \mathrm{~K}$

The rheological model used exhibits shear-thinning behavior but does not contemplate the existence of a yield stress, which is usually associated with blood. At very low shear rates, the RBCs form a three-dimensional solid-like structure suggesting that blood may have a yield stress. Increasing the shear stress, blood begins to flow and the solid-like structure ruptures. Nevertheless, the reported yield stress for blood is very small, ranging from $\sim 0.005$ to $\sim 0.01 \mathrm{~N} / \mathrm{m}^{2}$ [10] depending on the hematocrit and therefore, using a model without a yield stress is an acceptable approximation considering the high flow rate/shear rate conditions used in this work.

\section{Results}

\subsection{Newtonian Fluid Flow Patterns}

In this section, we present the results obtained with a Newtonian fluid (deionized water), flowing through the geometries described in Sect. 2.1. The investigation of the Newtonian fluid flow was carried out for comparison purposes.

Figures 4 and 5 show the flow patterns for two of the geometries studied $\left(S_{2}\right.$ and $S_{3}$, respectively) as a function of the Reynolds number, here defined as:

$$
R e=\frac{\rho V_{2} D_{2}}{\eta(\dot{\gamma})},
$$



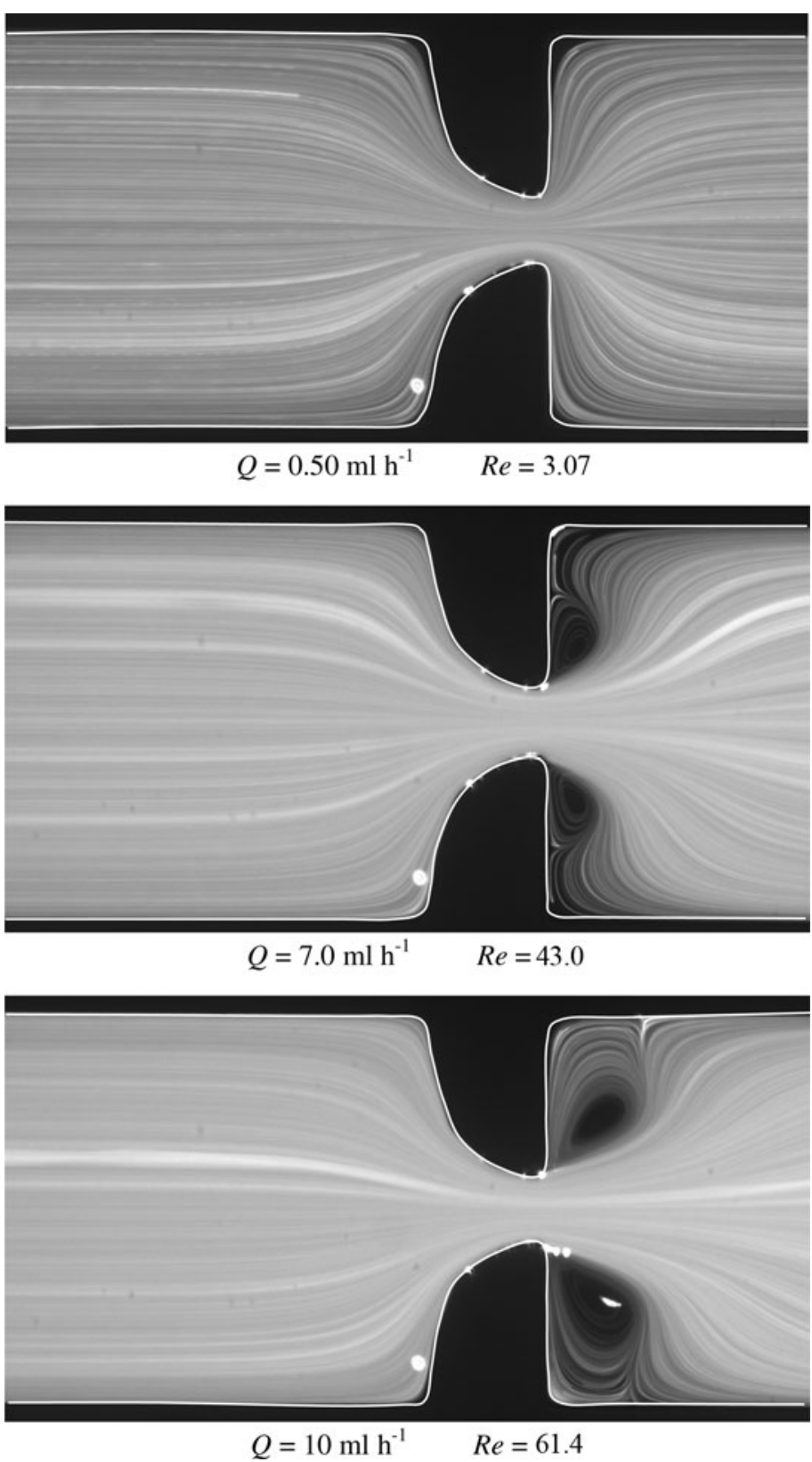

Fig. 4 Newtonian fluid flow patterns through the microchannel $S_{2}$, for different flow rates (or $R e$ ) 


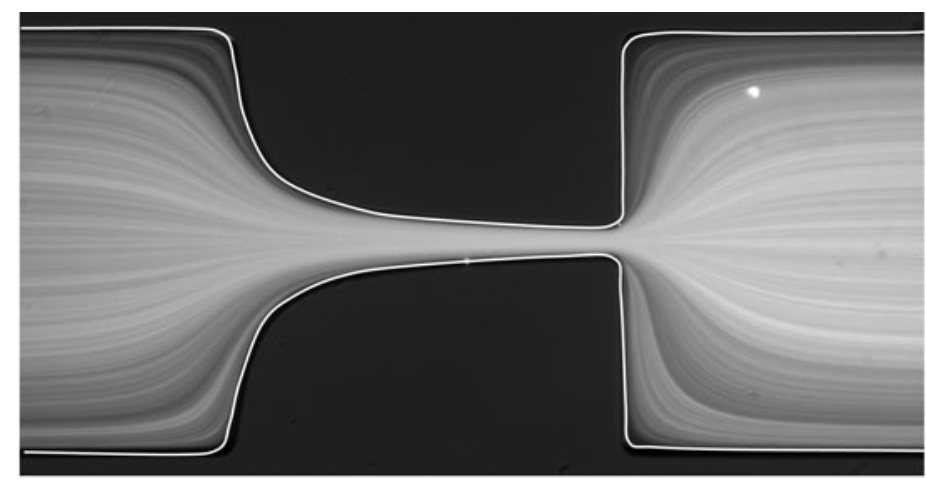

$$
Q=1.0 \mathrm{ml} \mathrm{h}^{-1} \quad R e=6.28
$$

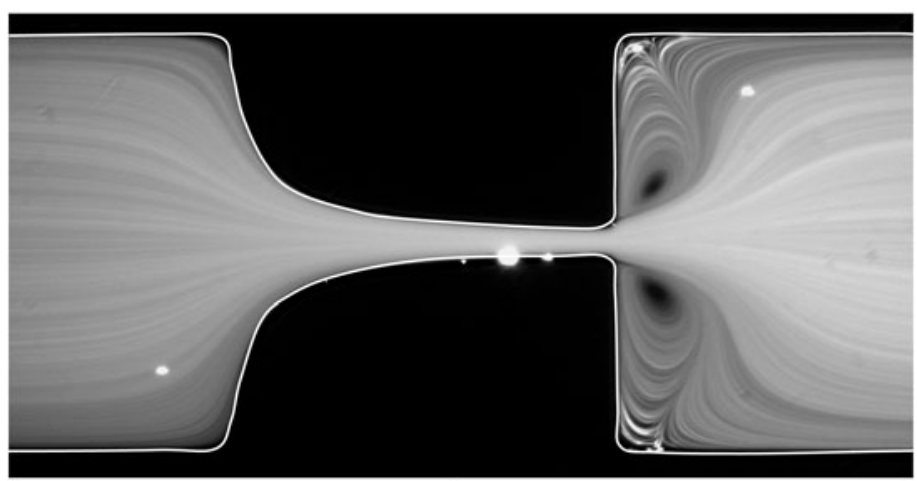

$$
Q=3.0 \mathrm{ml} \mathrm{h}^{-1} \quad \operatorname{Re}=18.9
$$

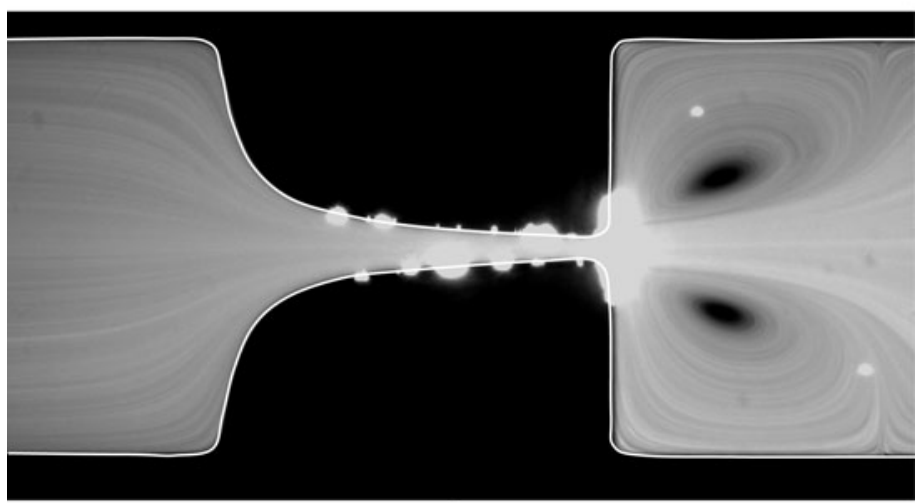

$$
Q=6.0 \mathrm{ml} \mathrm{h}^{-1} \quad R e=37.7
$$

Fig. 5 Newtonian fluid flow patterns through the microchannel $S_{3}$, for different flow rates (or $R e$ ) 
where $\rho$ is the density of the fluid and $V_{2}$ is the average velocity in the region of the contraction with width $D_{2}$. The shear viscosity is evaluated at a characteristic shear rate:

$$
\dot{\gamma}=\frac{V_{2}}{D_{2} / 2}
$$

and was calculated with the MPTT model using the parameters described in Sect. 2.3 for the viscoelastics fluid. For the Newtonian fluid the viscosity is constant.

For low $R e$, the fluid is pushed towards the centerline as it moves through the contraction and the flow remains attached to the wall downstream of the expansion, except near the far corner where a small weak separated flow region exists. An increase in the flow rate (and therefore in $R e$ ) leads to the onset of lip vortices close to the re-entrant corners downstream of the expansion plane. These vortices eventually enlarge to the far corner with increasing inertia of the flow.

For higher Hencky-strain geometries, the value of $R e$ at which the onset of lip vortices occurs is lower than for lower Hencky strains and the existing vortices tend to be elongated. Increasing inertia further, causes the vortices to grow in size and strength until a critical $R e$ is reached. At these critical conditions, the flow eventually becomes asymmetric with recirculations downstream of the expansion plane having different lengths (not illustrated), in agreement with the findings of Oliveira et al. [9] for abrupt contractions followed by abrupt expansions and Cherdron et al. [3] for expansions at the end of a channel.

\subsection{Viscoelastic Fluid Flow Patterns}

In Figs. 6 and 7 we show pathline images of the xanthan gum solution flowing through contraction-expansion geometries $S_{2}$ and $S_{3}$, respectively. The flow patterns observed for the viscoelastic fluid are qualitatively similar for all microgeometries studied, and only selected images are shown here. The following dimensionless parameter, the Deborah number, which represents the ratio between the relaxation time of the fluid, $\lambda$, and a characteristic time of the flow, $\left(D_{2} / 2\right) / V_{2}$, is also used in order to characterize the viscoelastic flow (cf. Fig. 1):

$$
D e=\frac{\lambda V_{2}}{D_{2} / 2} .
$$

At very low flow rates (and $D e$ ), the flow patterns are Newtonian-like and are not shown here for conciseness. Then, on increasing the flow rate, symmetric vortices develop upstream of the hyperbolic contraction due to the elasticity of the fluid. Increasing the Deborah number even further leads to an increase of the vortex size due to the enhancement of elastic effects. The formation of vortices close to the far corner upstream of the contraction has also been discussed by McKinley et al. [7] for flows of a $0.3 \mathrm{wt} \%$ PEO solution in a hyperbolic contraction followed by an abrupt expansion similar to the geometry used in this study. 


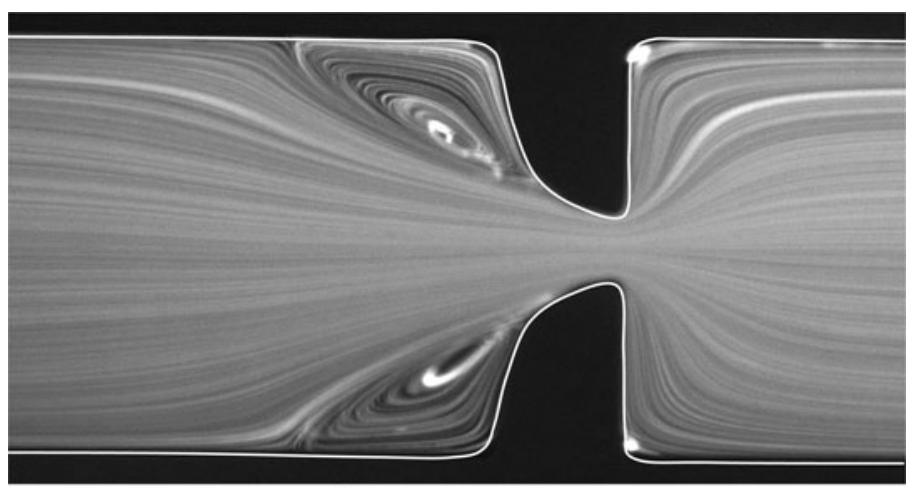

$$
Q=5.0 \mathrm{ml} \mathrm{h}^{-1} \quad R e=16.6 \quad D e=59.8
$$

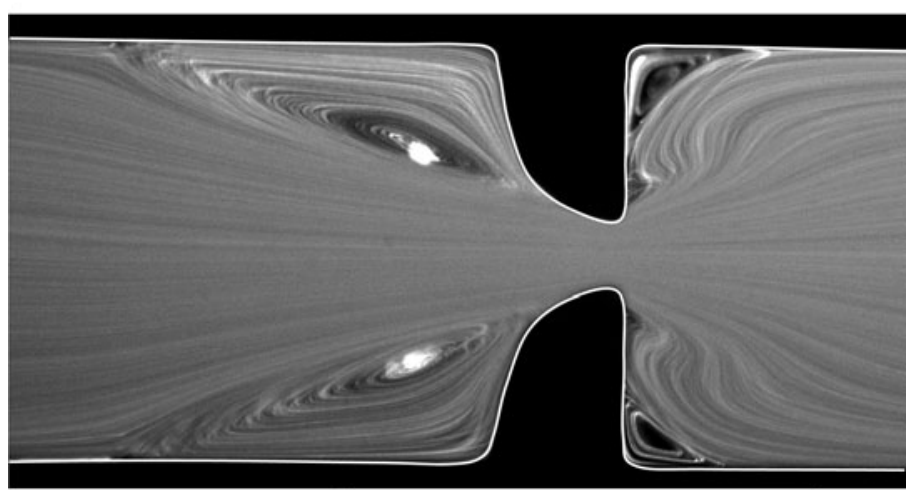

$$
Q=19 \mathrm{ml} \mathrm{h}^{-1} \quad R e=69.7 \quad D e=2.27 \times 10^{2}
$$

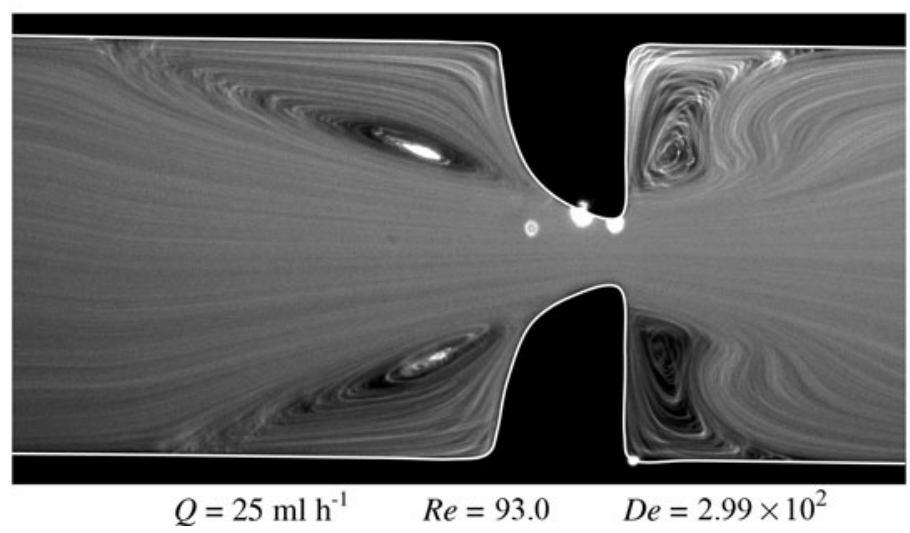

Fig. 6 Flow patterns of the xanthan gum solution in microchannel $S_{2}$ 


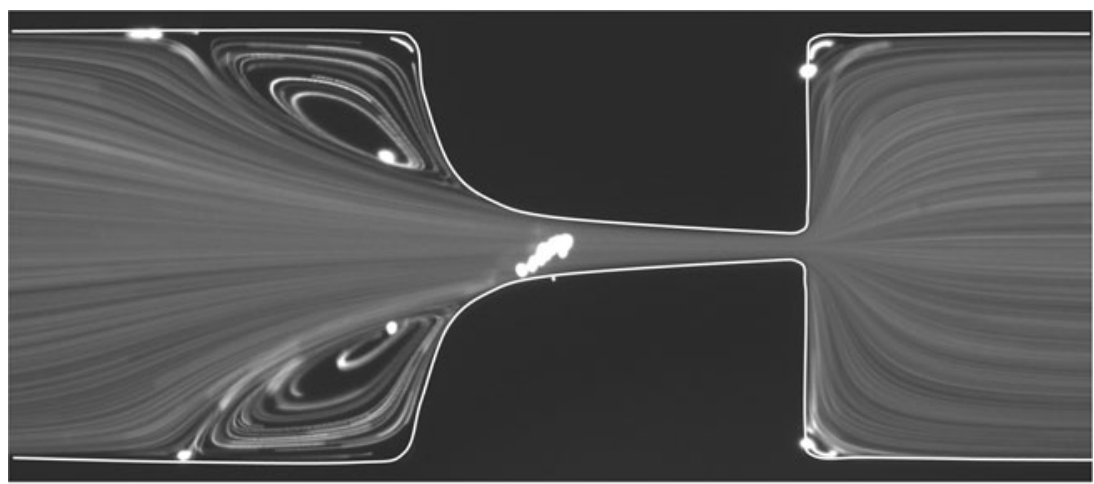

$$
Q=1.0 \mathrm{ml} \mathrm{h}^{-1} \quad R e=3.47 \quad D e=73.2
$$

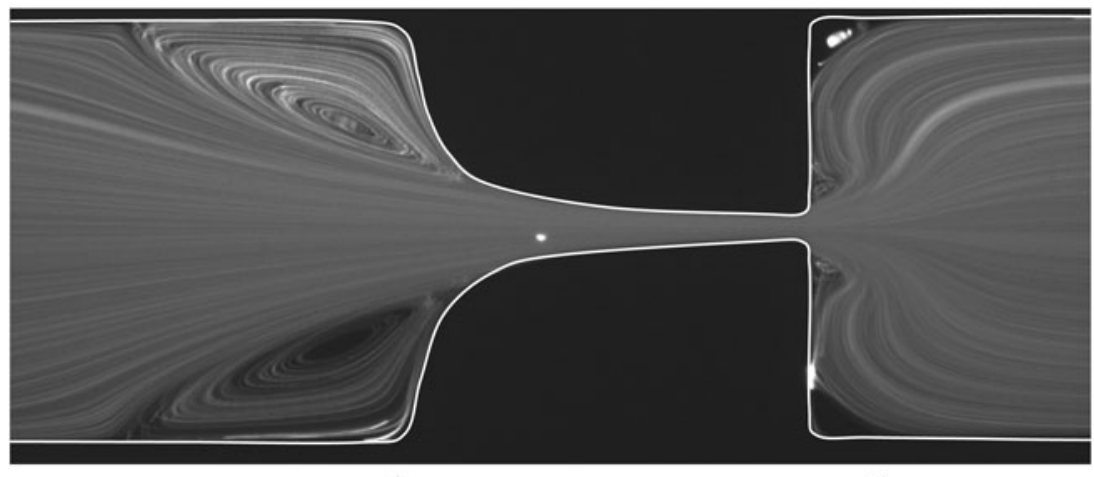

$$
Q=5.0 \mathrm{ml} \mathrm{h}^{-1} \quad R e=19.2 \quad D e=3.66 \times 10^{2}
$$

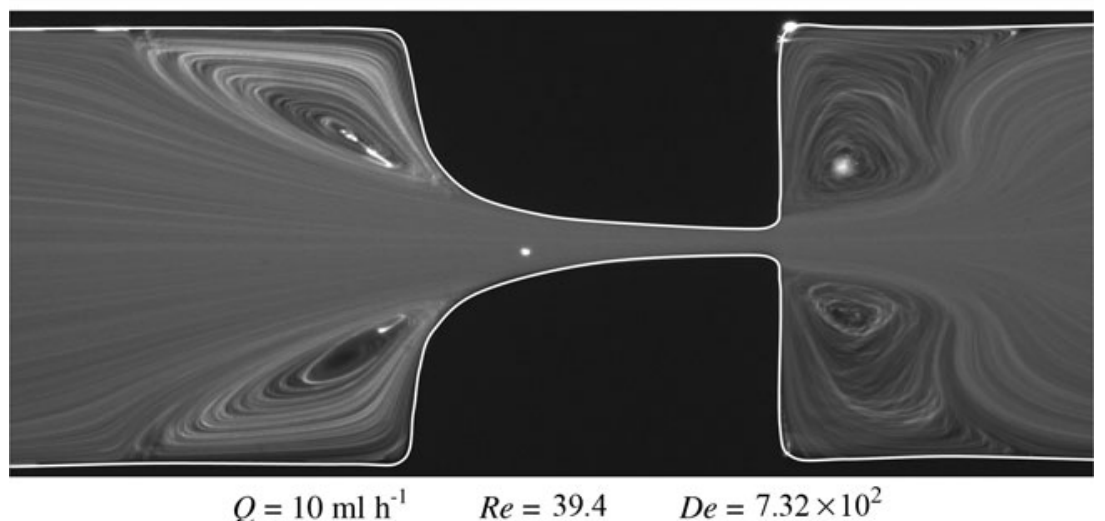

Fig. 7 Flow patterns of the xanthan gum solution in microchannel $S_{3}$ 
When the flow rate is increased even further, competing elastic and inertial effects are present and a lip vortex emerges downstream of the expansion plane as was observed for the Newtonian fluid flow when inertial effects become important. However, as a consequence of the relevance of elastic forces, in this case the inertiadriven downstream vortices are weaker than those observed for the Newtonian fluid at the same $R e$, since elasticity is responsible for the development of normal stresses in shear which are known to lead to jet swelling and consequently to a reduction of the size of downstream recirculations [12].

The small vortex at the far corner of the expansion also exists as for Newtonian fluids but again it is a rather weak entity. As the flow rate is further increased, the two recirculations downstream of the expansion merge into a single vortex that increases in size with the flow rate. Simultaneously, inertial effects start to predominate upstream, leading also to a decrease of the vortices located upstream of the hyperbolic contraction. At even higher flow rates, the flow becomes unstable with the upstream vortices varying in size asymmetrically along time. These results demonstrate that the blood analogue fluid used in this study shows viscoelastic behavior at the microscale, in spite of the small relaxation time. This enhancement of the viscoelastic behavior is particularly important at the microscale, and illustrates that whole human blood can possibly show important elastic effects when the characteristic dimension of the vessels decreases, as occurs in the microcirculation and even without considering the two-phase nature of blood. In the future, we expect to carry out similar experiments using real blood samples flowing in the microfluidic geometries used in this work, to probe whether blood indeed presents these viscoelastic flow features at the microscale.

Figure 8 summarizes our findings by mapping the flow patterns on the twodimensional $\varepsilon_{H}-D e$ parameter space. With this map, it is possible to foresee the different flow phenomena in the flow of a viscoelastic blood analogue solution through a constriction with different Hencky strains and Deborah numbers (notice the important presence of plane walls bounding the geometry): at low $D e$, symmetric vortices develop upstream of the contraction and on increasing flow rate, there is growth of the upstream vortices and the appearance of downstream corner vortices followed then by the appearance of downstream lip vortices and corner vortices growth. Then, merging of the downstream lip and corner vortices occurs and finally unstable flow is observed at even higher flow rates.

\section{Conclusions}

The flow of a viscoelastic blood analogue solution through microgeometries with a hyperbolic contraction followed by an abrupt expansion exhibits significantly different characteristics from those observed with a Newtonian fluid. In the latter, inertia promotes the appearance of vortices downstream of the abrupt expansion, while in the former complex flow patterns brought about by the elastic nature of the polymeric solution are observed. In particular, symmetric vortices appear upstream of the contraction due to elastic effects and when the flow rate is increased, inertial 

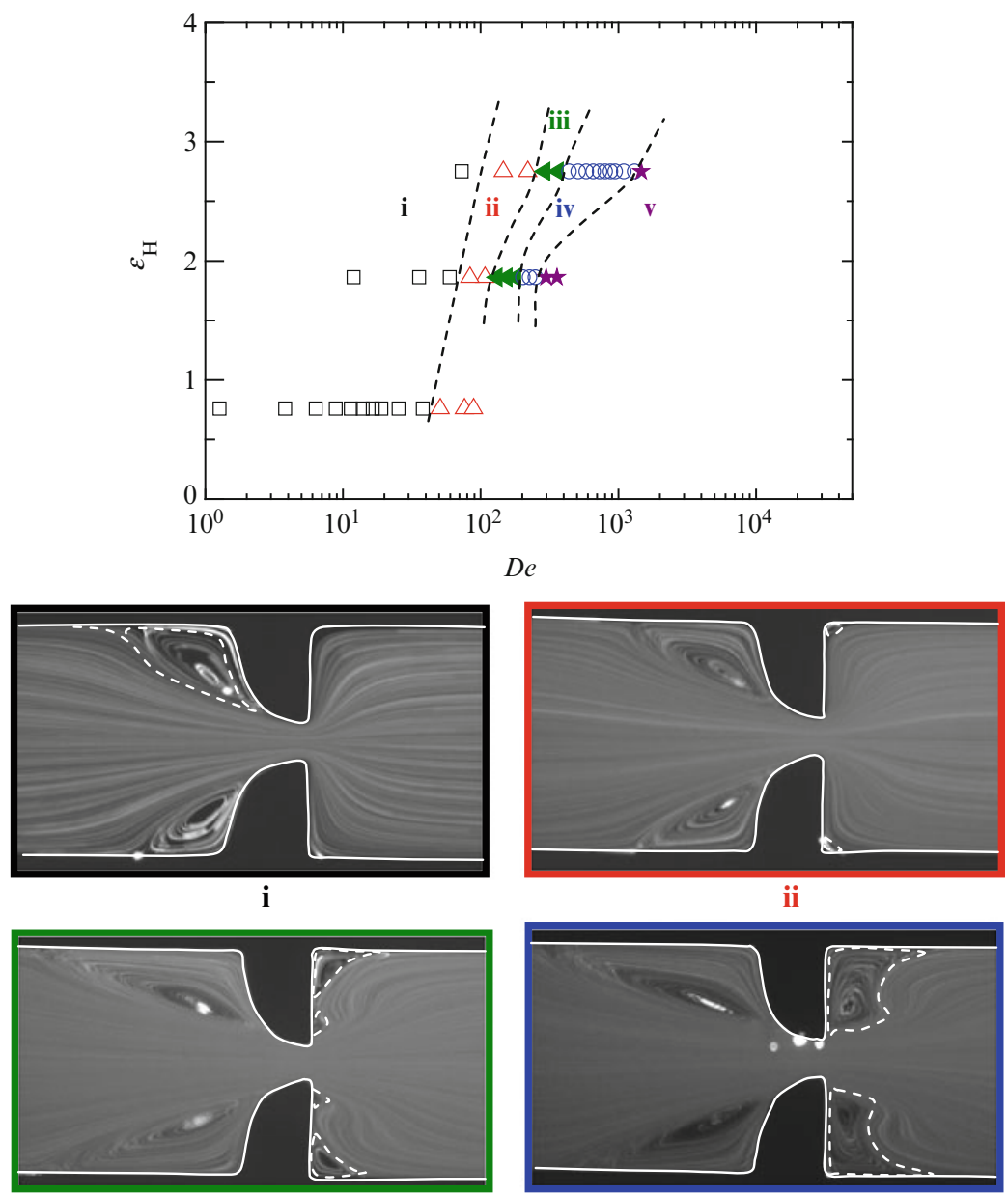

iii

iv

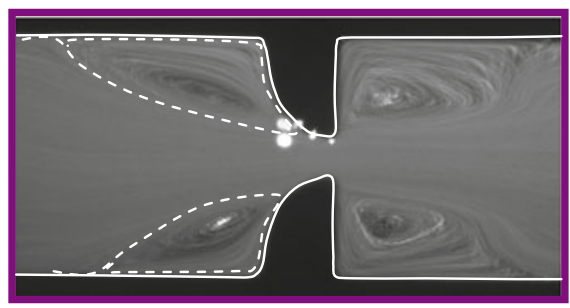

V

Fig. 8 Flow pattern map: (i) upstream symmetric vortices ( $\square$ ); (ii) downstream corner vortices $(\triangle)$; (iii) downstream lip and corner vortices ( $\triangle$ ); (iv) merging of downstream lip and corner vortices $(\bigcirc)$; (v) unstable (asymmetric) flow $(\star)$ 
effects lead to a decrease of the upstream vortices and the appearance and growth of downstream vortices. In addition, elasticity decreases the downstream vortices. These distinct behaviors clearly show that consideration of blood as a Newtonian fluid is a severe over-simplification that can prevent a correct prediction of blood flow behavior, especially in small sized vessels.

Acknowledgements The authors gratefully acknowledge funding by FCT via projects PTDC/EQU-FTT/71800/2006, REEQ/262/EME/2005, REEQ/928/EME/2005, PTDC/EMEMFE/099109/2008 and PTDC/EQU-FTT/70727/2006. In addition, PCS and ISP acknowledge the financial support of scholarships SFRH/BD/28846/2006 and CEFT/BII/2008/01.

\section{References}

1. Bird, R.B., Armstrong, R.C., Hassager O.: Dynamics of Polymeric Liquids. Volume 1: Fluid Dynamics Wiley, New York (1987)

2. Caro, C.G., Pedley, T.G., Seed, W.A.: Mechanics of the Circulation. Medical and Technical Publishers, London (1974)

3. Cherdron, W., Durst, F., Whitelaw, J.H.: Asymmetric flows and instabilities in symmetric ducts with sudden expansions. J Fluid Mech 84, 13-31 (1978)

4. Entov, V.M., Hinch, E.J.: Effect of a spectrum of relaxation times on the capillary thinning of a filament of elastic liquid. J Non-Newt Fluid Mech 72, 31-54 (1997)

5. Ku, D.N.: Blood flow in arteries. Annual Rev Fluid Mech 29, 399-434 (1997)

6. McDonald, J.C., Duffy, D.C., Anderson, J.R., et al.: Fabrication of microfluidic systems in poly(dimethylsiloxane). Electrophoresis 21, 27-40 (2000)

7. McKinley, G.H., Rodd, L.E., Oliveira, M.S.N., et al.: Extensional flows of polymeric solutions in microfluidic converging/diverging geometries. J Cent South Univ Technol 14, 6-9 (2007)

8. Oliveira, M.S.N., Alves, M.A., Pinho, F.T., et al.: Viscous flow through microfabricated hyperbolic contractions. Exp Fluids 43, 437-451 (2007)

9. Oliveira, M.S.N., Rodd, L.E., McKinley, G.H., et al.: Simulations of extensional flow in microrheometric devices. Microfluid Nanofluid 5, 809-826 (2008)

10. Owens, R.J.: A new microstructure-based constitutive model for human blood. J Non-Newt Fluid Mech 140, 57-70 (2006)

11. Phan-Thien, N., Tanner, R.I.: A new constitutive equation derived from network theory. J NonNewt Fluid Mech 2, 353-365 (1977)

12. Poole, R.J., Pinho, F.T., Alves, M.A., et al: The effect of expansion ratio for creeping expansion flows of UCM fluids. J Non-Newt Fluid Mech 163, 35-44 (2009)

13. Sdougos, H.P., Bussolari, S.R., Dewey, C.F.: Secondary flow and turbulence in a cone-and-plate device. J Fluid Mech 138, 379-404 (1984)

14. Thurston, G.B.: Viscoelastic properties of blood and blood analogs. Adv Hemodyn Hemorheol 1, 1-30 (1996)

15. Turitto, V.T.: Blood viscosity mass transport and thrombogenesis. Prog Hemost Thrombog 6, 139-177 (1982)

16. Vlastos, G., Lerche, D., Koch, B., et al.: The effect of parallel combined steady and oscillatory shear flows on blood and polymer solutions. Rheol Acta 36, 160-172 (1997)

17. Waite, L., Fine, J.: Applied Biofluid Mechanics. McGraw-Hill, New York (2007)

18. Xue, S.-C., Phan-Thien, N., Tanner, R.I. Numerical study of secondary flows of viscoelastic fluid in straight pipes by an implicit finite volume method. J Non-Newt Fluid Mech 59, 191-213 (1995)

19. Yilmaz, F., Gundogdu, M.Y.: A critical review on blood flow in large arteries; relevance to blood rheology, viscosity models, and physiologic conditions. Korea-Aust Rheol J 20, 197-211 (2008) 